\title{
Serum Asprosin Level in Different Subtypes of Polycystic Ovary Syndrome: A Cross-sectional Study
}

yonghui jiang

Shandong University https://orcid.org/0000-0003-1996-8954

yue liu

Shandong University

zhiheng yu

Shandong University

ping yang

Shandong University

lei xie

Shandong University

xuejun shang

Nanjing University

shigang zhao ( $\sim$ zsg0108@126.com )

Shandong University

Research

Keywords: asprosin, PCOS, hyperandrogenism, insulin resistance

Posted Date: July 17th, 2020

DOI: https://doi.org/10.21203/rs.3.rs-41704/v1

License: (c) (i) This work is licensed under a Creative Commons Attribution 4.0 International License.

Read Full License

Version of Record: A version of this preprint was published at Revista da Associação Médica Brasileira on April 1st, 2021. See the published version at https://doi.org/10.1590/1806-9282.20201147. 


\section{Title Page}

2 Title:

3 Serum asprosin level in different subtypes of polycystic ovary syndrome: a cross-sectional study

4 Author:

5 Yonghui Jiang ${ }^{1}$, Yue Liu ${ }^{1}$, Zhiheng Yu ${ }^{1}$, Ping Yang ${ }^{1}$, Lei Xie ${ }^{1}$, Xuejun Shang ${ }^{2}$, Shigang Zhao ${ }^{1 *}$

$6 \quad{ }^{1}$ Center for Reproductive Medicine, Cheeloo College of Medicine, Shandong University, Jinan

7 250001, China ${ }^{2}$ Department of Andrology, Jinling Hospital, School of Medicine, Nanjing

8 University, Nanjing 210002, China

$9 \quad{ }^{*}$ Correspondence should be addressed to Shigang Zhao(zsg0108@126.com)

\section{Corresponding author :}

12 Shigang Zhao, MD, Ph.D.

13 Center for Reproductive Medicine, Cheeloo College of Medicine, Shandong University

$14 \quad$ \#157 Jingliu Road, Jinan 250001, China

15 Tel: +86-18954109360

Email: zsg0108@126.com 


\section{Abstract}

Objective: Polycystic ovary syndrome (PCOS) can be divided into different subtypes, including insulin resistance (IR) and hyperandrogenism (HA). Asprosin is a novel hormone associated with IR; however, the role of asprosin in women with PCOS has not been investigated. Thus, the aim of this study was to investigate the relationship between serum asprosin levels and PCOS subtypes.

Methods: Ninety-three women with PCOS and 77 healthy women as controls were selected for this study. Clinical and laboratory data were compared between the PCOS group and the control group. The PCOS group was further divided into subgroups: 1) women with or without HA (PCOS HA and PCOS NHA, respectively); 2) women with or without IR (PCOS IR and PCOS NIR, respectively). Serum asprosin was measured by ELISA.

Results: Serum asprosin levels showed no significant difference between the PCOS and control groups. However, it was significantly lower in the PCOS HA and IR groups compared to the respective PCOS NHA and NIR groups $(\mathrm{P}<.05)$. In the PCOS group, serum asprosin was negatively correlated with body mass index, luteinizing hormone, testosterone, basal antral follicles, fasting insulin, Homeostatic Model Assessment of Insulin Resistance, and triglycerides. After adjusting for BMI, the correlations were not significant and asprosin was only positively correlated with prolactin $(\mathrm{r}=0.426, \mathrm{P}<.001)$.

Conclusions: Our study shows that women with PCOS HA or IR exhibit significantly lower serum asprosin levels compared to controls, and the lower asprosin level directly correlated with PRL level.

Keywords: asprosin; PCOS; hyperandrogenism; insulin resistance

\section{Background}


Polycystic ovary syndrome (PCOS), also known as Stein-Leventhal syndrome, is a common and complex endocrine metabolic disease caused by genetic and environmental factors. The prevalence is 5 to $10 \%$ in women of reproductive age[1]. PCOS is mainly characterized by menstrual abnormalities, infertility, hyperandrogenism (HA), polycystic ovarian morphology (PCOM), and metabolic abnormalities. Metabolic abnormalities are often manifested as obesity, insulin resistance (IR), and dyslipidemia[2, 3]. PCOS increases the risk for type 2 diabetes mellitus (T2DM), gestational diabetes, and other pregnancy-related complications, cardiovascular events, and endometrial cancer[4]. IR is considered as the major risk factor for the onset of PCOS[5] and 70\% of patients with PCOS have shown signs of IR[6].

Asprosin, a recently identified hormone, is secreted by the white adipose tissue (WAT) [7]. It is a 140-amino-acid fragment from the C-terminal of profibrillin (encoded by FBN1) and induces the liver to increase the levels of plasma glucose. Previous studies showed that asprosin was pathologically elevated in humans and mice with IR or obesity[7]. The olfactory receptor OLFR734 specifically binds with asprosin to modulate hepatic glucose production[8]. Several recent studies have shown that asprosin correlated with obesity in children and adults, T2DM and PCOS[9-16]. However, these results have been inconsistent. Thus, the aim of this study was to explore the potential relationship of asprosin with PCOS in women, especially those with HA or IR.

\section{Material and Methods}

\section{Study subjects}

The current study retrieved 170 serum samples, including 93 from the PCOS group and 77 from those without PCOS for the control group. The samples were obtained from the biobank affiliated to 
the Center for Reproductive Medicine of Shandong University. All serum samples were donated from infertility-related patients and were stored at $-80{ }^{\circ} \mathrm{C}$. PCOS was diagnosed by following the Rotterdam diagnostic criteria[17]: two of the following three criteria were positive, after the exclusion of other etiologies: 1) oligo and/or anovulation, 2) clinical and/or biochemical signs of hyperandrogenism, and 3) polycystic ovaries on ultrasonography. The exclusion criteria included women having androgen-secreting tumors, hyperprolactinemia, 21-hydroxylase deficiency (21-OHD), Cushing's syndrome, congenital adrenal hyperplasia, thyroid disease, or abnormal intrauterine cavity. A history of recurrent spontaneous abortion, intake of medications, antidiabetic drugs, antiandrogens, oral contraceptives, insulin sensitizers, glucocorticoids, and ovulation induction agents were also excluded. The threshold for defining PCOM on ultrasound was the presence of 12 or more follicles measuring 2-9 $\mathrm{mm}$ in diameter or an increased ovarian volume $(>10 \mathrm{~mL})$ in at least one ovary. The controls were age-matched women who had infertility related to male factors or tube factors, during the same period in our in vitro fertilization (IVF) program. PCOS was divided into subtypes according to testosterone (T) levels and homeostasis model of assessment for insulin resistance index (HOMAIR): PCOS with HA (PCOS HA, T > 60 ng/dL) and without HA (PCOS NHA); PCOS with IR (PCOS IR, HOMA-IR $\geqslant 2.5$ ) and without IR (PCOS NIR) $[18,19]$. All the serum samples were collected in the follicular phase. Venous blood samples were collected in the morning after an overnight fasting period.

\section{Clinical and laboratory data collection}

Clinical and laboratory data were collected from electronic medical records (EMR) in our hospital. Anthropometric data included height, weight, body mass index (BMI), and menstrual cycle history. Serum hormones measured included follicle stimulating hormone (FSH), luteinizing hormone (LH), 
estradiol (E2), prolactin (PRL), testosterone (T), thyroid-stimulating hormone (TSH), dehydroepiandrosterone sulfate (DHEA-S), and anti-müllerian hormone (AMH); these were tested using electrochemiluminescence. Basal antral follicles were counted between the third day and fifth day of menstruation by vaginal ultrasonic examination. Metabolic related indicators including fasting glucose, fasting insulin, total cholesterol (TC), high-density lipoprotein cholesterol (HDL-C), lowdensity lipoprotein cholesterol (LDL-C), and triglycerides (TG) were measured. HOMA-IR was calculated as fasting glucose (in mmol/L) * fasting insulin (in $\mathrm{mIU} / \mathrm{L}) / 22.5$.

\section{Measurement of asprosin}

Serum asprosin was measured using a commercial human asprosin ELISA Kit (Catalog No: E15190h, Wuhan EIAab Science Co. Ltd., China) according to the manufacturer's instructions. In brief, $100 \mu \mathrm{L}$ serum covered with the plate sealer was incubated at $37^{\circ} \mathrm{C}$ for 2 hours. The liquid was then removed and $100 \mu \mathrm{L}$ of detection reagent $\mathrm{A}$ was added and incubated at $37^{\circ} \mathrm{C}$ for 1 hour. Next, the sample was washed 3 times and $100 \mu \mathrm{L}$ detection reagent B was added. After 1-hour incubation, the sample was washed 5 times and $90 \mu \mathrm{L}$ of substrate solution was added. A $50 \mu \mathrm{L}$ stop solution was then added and $450 \mathrm{~nm}$ optical density was determined by automated microplate reader (PerkinElmer, Inc., Waltham, MA, USA). Acquired data were calculated by CurveExpert 1.4 (Hyams D.G., Starkvile, MS.USA). The intra-assay coefficient of variation (CV) was $\leq 6.5 \%$ and the inter-assay $\mathrm{CV}$ was $\leq$ $9.8 \%$.

\section{Statistical analysis}

All statistical analyses were conducted using SPSS software (IBM, Armonk, NY, version 21.0) and GraphPad Prism 7 software (San Diego, CA, USA). Kolmogorov-Smimov test were used to test characteristics of participants' distribution. Data normally distributed were expressed as mean \pm SD 
and data with skewed distribution were shown as median (IQR, 25th-75th). Independent samples ttest was used to compare the normally distributed variables and Man-Whitney U test was used to compare abnormally distributed variables. Spearman correlation analysis was performed to analyze bivariate correlation between asprosin and other parameters. $\mathrm{P}$ value $<.05$ (two-sided) was considered as statistically significant. The sample size power calculation was performed from an online website (http://powerandsamplesize.com/). Considering the score between PCOS and control subjects (power $>0.8 ; \alpha=0.05$; sampling ratio $=2$ ), 802 PCOS cases and 1604 control cases were required. While considering the score between PCOS subtypes (power $>0.8 ; \alpha=0.05$; sampling ratio =1), 41 PCOS HA cases and 56 PCOS IR cases were required.

\section{Results}

\section{Characteristics of the clinical subjects}

Clinical characteristics of the 170 subjects are described in Table 1 . There were no significant differences in age, FSH, PRL, and TSH levels between the control and PCOS groups. PCOS patients had higher levels of different hormones and metabolic-associated parameters (LH, LH/FSH, E2, T, AMH, fasting glucose, fasting insulin, HOMA-IR, DHEA-S, TC, HDL,LDL, and TG, $\mathrm{P}<.05$ ). Women in the PCOS group had significantly longer menstrual cycles than those in the control group $(50.87 \pm 13.68$ vs $29.69 \pm 2.98 ; \mathrm{P}<.001)$. In the PCOS group, BMI was higher and basal antral follicle numbers were more than in the control group $(\mathrm{P}<.05)$.

\section{Serum asprosin levels in distinct groups}

As shown in Fig.1A, serum asprosin levels showed no significant difference between the PCOS and control groups [2.87 (2.18-4.47) vs. $3.24(2.23-4.31) \mathrm{ng} / \mathrm{mL}$, median(25th-75th), $\mathrm{P}>\quad .05]$. The 
asprosin levels were measured in different PCOS subtypes (Supplementary Table1, Supplementary Table2 and Fig.1B-D). Serum asprosin level in the PCOS HA group was notably lower than in the PCOS NHA group [2.52 (2.06-3.19) vs. 4.20 (2.35-5.79) ng/mL, median (25th-75th), $\mathrm{P}<.05]$ (Fig.1B). Serum asprosin levels in the PCOS IR group were significantly lower than in the PCOS NIR group [2.46 (2.05-4.30) vs. 3.77 (2.47-7.18) ng/mL, median (25th-75th), $\mathrm{P} \quad$.05] (Fig.1C). In addition, this trend was more pronounced in the PCOS HA \& IR groups.

\section{Correlation coefficient of variables associated with circulating asprosin}

As listed in Table 2, Spearman analysis indicated that serum asprosin was positively correlated with TSH and HDL, and negatively correlated with BMI, fasting insulin, HOMA-IR, and TG in all the samples. However, after adjustment for BMI, asprosin was positively correlated only with PRL (r $=0.399, \mathrm{P}<.001)$, TSH $(\mathrm{r}=0.162, \mathrm{P}=.038)$, and HDL $(\mathrm{r}=0.178, \mathrm{P}=.033)$. While in the PCOS group, serum asprosin was negatively correlated with BMI, LH, T, basal antral follicles, fasting insulin, HOMA-IR, and TG. When adjusted for BMI, the correlations were not significant and asprosin was only positively correlated with PRL $(\mathrm{r}=0.426, \mathrm{P}<.001$; Table 3$)$. In addition, asprosin was still positively correlated with PRL $(r=0.456, \mathrm{P}=.003)$ in PCOS NHA subjects. Moreover, there was no correlation between asprosin and other characteristics in PCOS HA subjects. These results indicate that obesity rather than PCOS might be responsible for the difference in asprosin levels.

\section{Discussion}

The current study showed that serum asprosin levels were similar between women in PCOS and control groups; however, lower levels were seen in PCOS HA and PCOS IR groups. Further analysis demonstrated that asprosin was positively correlated with PRL, independent of BMI. 

IR[7]. Although IR was excluded from the diagnostic criteria for PCOS, it is a common physiological abnormality with metabolic dysfunctions in women with PCOS[20]. Adipose tissue can regulate the metabolism and balance the energy homeostasis through its role in endocrine regulation. Some small molecules secreted by the adipose tissue can either enhance or impair insulin action[21, 22]. Moreover, Romere and his colleagues verified that asprosin level was higher in humans and mice with IR[7].

Recently, two other groups confirmed that asprosin was positively correlated with diabetes mellitus[16, 23]. There have been reports about the relationship between asprosin and PCOS; however, these results are inconsistent $[13,15,24]$. We would like to further explore the profiles of asprosin in PCOS subtypes, and HA is one of the most important phenotypes of PCOS.

We first compared the serum asprosin in women with PCOS and healthy women in the concurrent period. Serum asprosin was comparable between women with or without PCOS, and it was somewhat lower in the PCOS group. The PCOS group was then divided into different subgroups. Serum asprosin levels were lower in both, PCOS HA and IR groups, which was contrary to our expectations. We then analyzed the probable correlations between asprosin and PCOS. The results showed that asprosin was negatively correlated with IR and HOMA-IR, which contradicts previous studies[7, 16, 23]. However, after adjusting for BMI, there was a positive correlation only between asprosin and PRL. Therefore, it is likely that obesity rather than PCOS might be responsible for the difference in asprosin levels. There might be some explanations for this. One possibility is that the serum asprosin might be influenced by confounding effects through sex hormones, specific population conditions, and repeated freeze-thaw cycles. Another possibility is the multiple interactions with some other adipokines such as irusin, visfatin, and adiponectin, which are also secreted by the white adipose 
tissue (WAT) and associated with PCOS[25-27]. Meanwhile, we found that asprosin was positively correlated with PRL. Circulating PRL is mainly secreted by the lactotroph and mammosomatotroph cells in the pituitary gland. However, the adipose tissue can also produce PRL at extra-pituitary sites[28]. In both, young healthy men[29] and overweight or obese men[30], PRL was inversely associated with insulin sensitivity. PRL produced by the adipose tissue was directly related to the PPARG, ADIPOQ and GLUT4 levels in the human visceral and subcutaneous fat[30]. Considered together, PRL might influence asprosin levels through certain feedback mechanisms in women with PCOS, which also explains the first possibility. Moreover, elevated levels of insulin can stimulate ovarian androgen production and cause elevated $\mathrm{T}$ by suppressing the sex-hormone binding globulin (SHBG) [31]. We did not find any further correlations between asprosin levels and $\mathrm{T}$ after adjusting for BMI. The specific mechanism might be beyond our cognition in this limited study and more indepth research is needed.

Our study provides significant insights about the correlation between asprosin and PCOS. Three different studies about asprosin and PCOS were recently published[13, 15, 24]. Chia et al reported that asprosin levels in women with PCOS were similar to those in corresponding controls[24]. However, Murat and Li found that circulating asprosin levels were elevated in women with PCOS compared to those in controls[13, 15]. Our results were consistent with former, but contrary to the latter. This diversity might be due to the different sample conditions and effects of PRL. Yet, some limitations in this study should be acknowledged. For example, the study is based on EMR from a single hospital and the sample size was relatively limited.

\section{Conclusions}

This study shows that women with PCOS HA or IR exhibit significantly lower levels of serum 
asprosin. The serum asprosin levels also correlated closely with various sex hormones and metabolic disorders, and the lower asprosin levels directly correlated with PRL levels. Our research provides new clinical insights about the role of adipokines in the pathogenesis of PCOS.

\section{Acknowledgments}

We thank Rusong Zhao and Wei Zhou for their statistical analysis. We also thank Wolters Kluwer for providing language polish services.

\section{Author contributions}

YH Jiang: data curation, project administration, validation, writing (original draft). Y Liu and ZH Yu: serum sample collection, ELISA test performing. P Yang and L Xie: clinical data collection, data analysis. XJ Shang: investigation, methodology, supervision, validation. SG Zhao: funding acquisition, investigation, methodology, software, supervision, validation, visualization, writing (review/editing).

\section{Funding}

This study was funded by National Key Research and Development Program of China (2018YFC1004303), and Young Scholars Program of Shandong University.

\section{Availability of data and materials}

All of the related results can be requested from the corresponding author.

\section{Ethics approval and consent to participate}

This study involving human serum samples and clinical data were approved by the ethics committee of Center for Reproductive Medicine of Shandong University.

\section{Consent for publication}


Not applicable.

\section{Competing interests}

223 The authors declare that there is no conflict of interest regarding the publication of this paper.

\section{Acknowledgments}

We thank Rusong Zhao and Wei Zhou for their statistical analysis. We also thank Wolters Kluwer for providing language polish services.

\section{Authors' details}

\section{References}


Ranasinha S, Teede HJ, Moran LJ. Ethnicity, obesity and the prevalence of impaired glucose tolerance and type 2 diabetes in PCOS: a systematic review and meta-regression. Hum Reprod Update. 2018;24(4):455-67.

4. Azziz R, Carmina E, Chen Z, Dunaif A, Laven JS, Legro RS, Lizneva D, Natterson-Horowtiz B, Teede HJ, Yildiz BO. Polycystic ovary syndrome. Nat Rev Dis Primers. 2016;2:16057.

5. Diamanti-Kandarakis E, Dunaif A. Insulin resistance and the polycystic ovary syndrome

6. DeUgarte CM, Bartolucci AA, Azziz R. Prevalence of insulin resistance in the polycystic

9. Wang M, Yin C, Wang L, Liu Y, Li H, Li M, Yi X, Xiao Y. Serum Asprosin Concentrations

8. Li E, Shan H, Chen L, Long A, Zhang Y, Liu Y, Jia L, Wei F, Han J, Li T, Liu X, Deng H, Wang Y. OLFR734 Mediates Glucose Metabolism as a Receptor of Asprosin. Cell Metab. 2019;30(2):319-28.e8.

11. Long W, Xie X, Du C, Zhao Y, Zhang C, Zhan D, Li Z, Ning Q, Luo X. Decreased Circulating 
Levels of Asprosin in Obese Children. Horm Res Paediatr. 2019;91(4):271-7.

12. Ugur K, Aydin S. Saliva and Blood Asprosin Hormone Concentration Associated with Obesity. Int J Endocrinol. 2019;2019:2521096.

13. Li X, Liao M, Shen R, Zhang L, Hu H, Wu J, Wang X, Qu H, Guo S, Long M, Zheng H. Plasma Asprosin Levels Are Associated with Glucose Metabolism, Lipid, and Sex Hormone Profiles in Females with Metabolic-Related Diseases. Mediators Inflamm. 2018;2018:7375294.

14. Wang CY, Lin TA, Liu KH, Liao CH, Liu YY, Wu VC, Wen MS, Yeh TS. Serum asprosin levels and bariatric surgery outcomes in obese adults. Int J Obes (Lond). 2019;43(5):1019-25.

15. Alan M, Gurlek B, Yilmaz A, Aksit M, Aslanipour B, Gulhan I, Mehmet C, Taner CE. Asprosin: a novel peptide hormone related to insulin resistance in women with polycystic ovary syndrome. Gynecol Endocrinol. 2019;35(3):220-3.

16. Zhang L, Chen C, Zhou N, Fu Y, Cheng X. Circulating asprosin concentrations are increased in type 2 diabetes mellitus and independently associated with fasting glucose and triglyceride. Clin Chim Acta. 2019;489:183-8.

17. Rotterdam ESHRE/ASRM-Sponsored PCOS Consensus Workshop Group. Revised 2003 consensus on diagnostic criteria and long-term health risks related to polycystic ovary syndrome. Fertil Steril. 2004;81(1):19-25.

18. Cui Y, Shi Y, Cui L, Han T, Gao X, Chen ZJ. Age-specific serum antimüllerian hormone levels in women with and without polycystic ovary syndrome. Fertil Steril. 2014;102(1):230-6.e2.

19. Andrade MI, Oliveira JS, Leal VS, Lima NM, Costa EC, Aquino NB, Lira PI. [Identification of cutoff points for Homeostatic Model Assessment for Insulin Resistance index in 
adolescents: systematic review]. Rev Paul Pediatr. 2016;34(2):234-42.

20. Escobar-Morreale HF. Polycystic ovary syndrome: definition, aetiology, diagnosis and treatment. Nat Rev Endocrinol. 2018;14(5):270-84.

21. Andrade-Oliveira V, Câmara NO, Moraes-Vieira PM. Adipokines as drug targets in diabetes and underlying disturbances. J Diabetes Res. 2015;2015:681612.

22. Booth A, Magnuson A, Fouts J, Foster MT. Adipose tissue: an endocrine organ playing a role in metabolic regulation. Horm Mol Biol Clin Investig. 2016;26(1):25-42.

23. Wang Y, Qu H, Xiong X, Qiu Y, Liao Y, Chen Y, Zheng Y, Zheng H. Plasma Asprosin Concentrations Are Increased in Individuals with Glucose Dysregulation and Correlated with Insulin Resistance and First-Phase Insulin Secretion. Mediators Inflamm. 2018;2018:9471583.

24. Chang CL, Huang SY, Hsu YC, Chin TH, Soong YK. The serum level of irisin, but not asprosin, is abnormal in polycystic ovary syndrome patients. Sci Rep. 2019;9(1):6447.

25. Zhang L, Fang X, Li L, Liu R, Zhang C, Liu H, Tan M, Yang G. The association between circulating irisin levels and different phenotypes of polycystic ovary syndrome. J Endocrinol Invest. 2018;41(12):1401-7.

26. Tsouma I, Kouskouni E, Demeridou S, Boutsikou M, Hassiakos D, Chasiakou A, Hassiakou S, Baka S. Correlation of visfatin levels and lipoprotein lipid profiles in women with polycystic ovary syndrome undergoing ovarian stimulation. Gynecol Endocrinol. 2014;30(7):516-9.

27. Benrick A, Chanclón B, Micallef P, Wu Y, Hadi L, Shelton JM, Stener-Victorin E, Wernstedt Asterholm I. Adiponectin protects against development of metabolic disturbances in a PCOS mouse model. Proc Natl Acad Sci U S A. 2017;114(34):E7187-7187E7196. 
28. Ben-Jonathan N, Hugo E. Prolactin (PRL) in adipose tissue: regulation and functions. Adv Exp Med Biol. 2015;846:1-35.

29. Wagner R, Heni M, Linder K, Ketterer C, Peter A, Böhm A, Hatziagelaki E, Stefan N, Staiger H, Häring HU, Fritsche A. Age-dependent association of serum prolactin with glycaemia and insulin sensitivity in humans. Acta Diabetol. 2014;51(1):71-8.

30. Wang T, Lu J, Xu Y, Li M, Sun J, Zhang J, Xu B, Xu M, Chen Y, Bi Y, Wang W, Ning G. Circulating prolactin associates with diabetes and impaired glucose regulation: a populationbased study. Diabetes Care. 2013;36(7):1974-80.

31. Glintborg D, Petersen MH, Ravn P, Hermann AP, Andersen M. Comparison of regional fat mass measurement by whole body DXA scans and anthropometric measures to predict insulin resistance in women with polycystic ovary syndrome and controls. Acta Obstet Gynecol Scand. 2016;95(11):1235-43. 


\section{Figure Legends:}

Fig.1. Serum asprosin levels between distinct groups. (A) Serum asprosin in PCOS group (n=89) had no significant statistically difference compared to the control group $(\mathrm{n}=75)$ [P $>0.05]$. (B) PCOS was 328 subdivided into hyperandrogenism subtype (PCOS HA, $n=47$ ) and none-hyperandrogenism subtype (PCOS NHA, $n=42$ ). Serum asprosin in PCOS HA group were significantly lower than the PCOS

NHA group $(\mathrm{P}<0.05)$. (C) PCOS patients were subdivided into insulin resistance subtype (PCOS IR, $\mathrm{n}=46$ ) and none-insulin resistance subtype (PCOS NIR, $n=34$ ). Serum asprosin in PCOS IR group were significantly lower than the PCOS NIR group (P < 0.05). (D) PCOS patients with both IR and HA (PCOS IR\&HA, $n=35) . *$ indicates statistical significance at $\mathrm{p}<0.05$ and $* *$ indicates statistical significance at $\mathrm{p}<0.01$.

Additional files:

Supplementary Table 1 .docx

General clinical and laboratory characteristics of PCOS NHA and PCOS HA subjects

\section{Supplementary Table 2 .docx}

General clinical and laboratory characteristics of PCOS NIR and PCOS IR subjects 

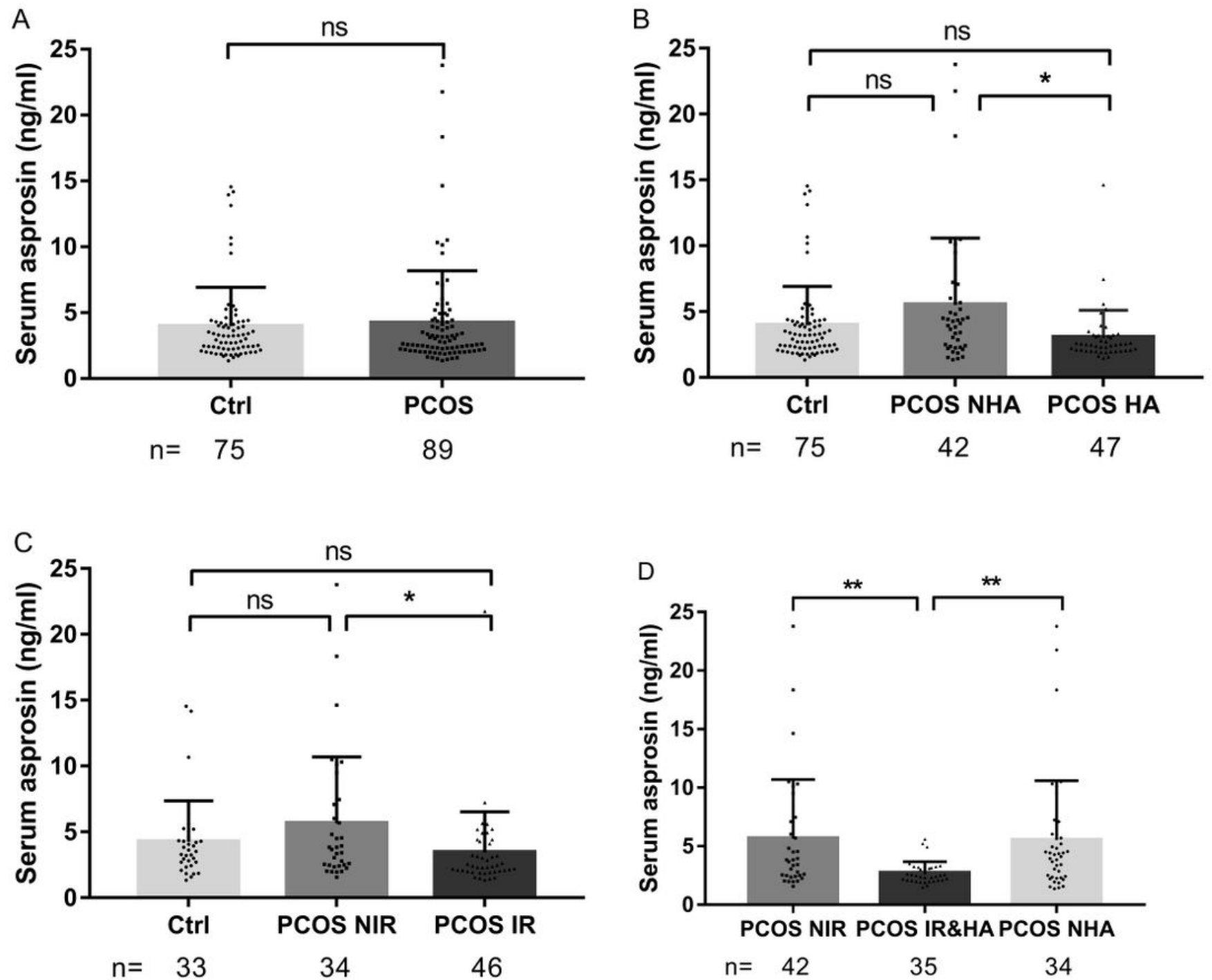

\section{Figure 1}

Serum asprosin levels between distinct groups. (A) Serum asprosin in PCOS group $(n=89)$ had no significant statistically difference compared to the control group $(n=75)$ पPख0.05区. (B) PCOS was subdivided into hyperandrogenism subtype (PCOS HA, n=47) and none-hyperandrogenism subtype (PCOS NHA, $n=42$ ). Serum asprosin in PCOS HA group were significantly lower than the PCOS NHA group (Pख0.05). (C) PCOS patients were subdivided into insulin resistance subtype (PCOS IR, n=46) and none-insulin resistance subtype (PCOS NIR, $n=34$ ). Serum asprosin in PCOS IR group were significantly lower than the PCOS NIR group (P凶0.05). (D) PCOS patients with both IR and HA (PCOS IR\&HA, n=35). * indicates statistical significance at $p<0.05$ and $* \star$ indicates statistical significance at $p<0.01$.

\section{Supplementary Files}


This is a list of supplementary files associated with this preprint. Click to download.

- SupplementaryTable2.docx

- SupplementaryTable1.docx

- Tables.pdf 